\title{
SETS WITH ALMOST COINCIDING REPRESENTATION FUNCTIONS
}

\author{
SÁNDOR Z. KISS ${ }^{凶}$, ESZTER ROZGONYI and CSABA SÁNDOR
}

(Received 20 November 2012; accepted 15 April 2013; first published online 28 June 2013)

\begin{abstract}
For a given integer $n$ and a set $\mathcal{S} \subseteq \mathbb{N}$, denote by $R_{h, S}^{(1)}(n)$ the number of solutions of the equation $n=s_{i_{1}}+\cdots+s_{i_{h}}, s_{i_{j}} \in \mathcal{S}, j=1, \ldots, h$. In this paper we determine all pairs $(\mathcal{A}, \mathcal{B}), \mathcal{A}, \mathcal{B} \subseteq \mathbb{N}$, for which $R_{3, \mathcal{A}}^{(1)}(n)=R_{3, \mathcal{B}}^{(1)}(n)$ from a certain point on. We discuss some related problems.

2010 Mathematics subject classification: primary 11B34.

Keywords and phrases: additive number theory, representation functions.
\end{abstract}

\section{Introduction}

Let $\mathbb{N}$ be the set of nonnegative integers. For a given infinite set $\mathcal{A} \subset \mathbb{N}$ the representation functions $R_{h, \mathcal{A}}^{(1)}(n), R_{h, \mathcal{A}}^{(2)}(n)$ and $R_{h, \mathcal{A}}^{(3)}(n)$ are defined in the following way:

$$
\begin{aligned}
& R_{h, \mathcal{A}}^{(1)}(n)=\#\left\{\left(a_{i_{1}}, \ldots, a_{i_{h}}\right): a_{i_{1}}+\cdots+a_{i_{h}}=n, a_{i_{1}}, \ldots, a_{i_{h}} \in \mathcal{A}\right\}, \\
& R_{h, \mathcal{A}}^{(2)}(n)=\#\left\{\left(a_{i_{1}}, \ldots, a_{i_{h}}\right): a_{i_{1}}+\cdots+a_{i_{h}}=n, a_{i_{1}}, \ldots, a_{i_{h}} \in \mathcal{A}, a_{i_{1}} \leq \cdots \leq a_{i_{h}}\right\}, \\
& R_{h, \mathcal{A}}^{(3)}(n)=\#\left\{\left(a_{i_{1}}, \ldots, a_{i_{h}}\right): a_{i_{1}}+\cdots+a_{i_{h}}=n, a_{i_{1}}, \ldots, a_{i_{h}} \in \mathcal{A}, a_{i_{1}}<\cdots<a_{i_{h}}\right\} .
\end{aligned}
$$

Representation functions have been extensively studied by many authors and are still a fruitful area of research in additive number theory. Using generating functions, Nathanson [6] proved the following result.

Let $A, B$ and $T$ be finite sets of integers. If each residue class modulo $m$ contains exactly the same number of elements of $A$ as elements of $B$, then we write $A \equiv B$ $(\bmod m)$. If the number of solutions of the congruence $a+t \equiv n(\bmod m)$ with $a \in A$, $t \in T$, equals the number of solutions of the congruence $b+t \equiv n(\bmod m)$ with $b \in B$, $t \in T$, for each residue class $n$ modulo $m$, then we write $A+T \equiv B+T(\bmod m)$.

The first author was supported by OTKA Grant No. K77476 and No. NK105645. The work reported in this paper was carried out in the framework of the 'Talent care and cultivation in the scientific workshops of BME' project. This project is supported by grant TáMOP - 4.2.2.B-10/1-2010-0009. The third author was supported by OTKA Grant No. K81658.

(C) 2013 Australian Mathematical Publishing Association Inc. 0004-9727/2013 \$16.00 
Nathanson's Theorem. Let $\mathcal{A}$ and $\mathcal{B}$ be infinite sets of nonnegative integers, $\mathcal{A} \neq \mathcal{B}$. Then $R_{2, \mathcal{A}}^{(1)}(n)=R_{2, \mathcal{B}}^{(1)}(n)$ from a certain point on if and only if there exist positive integers $N$, $m$ and finite sets $A, B, T$ with $A \cup B \subset\{0,1, \ldots, N\}$ and $T \subset\{0,1, \ldots, m-1\}$ such that $A+T \equiv B+T(\bmod m)$, and $\mathcal{A}=A \cup C$ and $\mathcal{B}=B \cup C$, where $C=\{c>N: c \equiv t$ $(\bmod m)$ for some $t \in T\}$.

It is clear that $R_{2, \mathcal{A}}^{(2)}(n)=\left\lceil R_{2, \mathcal{A}}^{(1)}(n) / 2\right\rceil$ and $R_{2, \mathcal{A}}^{(3)}(n)=\left\lfloor R_{2, \mathcal{A}}^{(1)}(n) / 2\right\rfloor$, so for the sets $\mathcal{A}, \mathcal{B}$ in Nathanson's theorem we have $R_{2, \mathcal{A}}^{(2)}(n)=R_{2, \mathcal{B}}^{(2)}(n)$ and $R_{2, \mathcal{A}}^{(3)}(n)=R_{2, \mathcal{B}}^{(3)}(n)$ from a certain point on. It is easy to see that the symmetric difference of the sets $\mathcal{A}$ and $\mathcal{B}$ in the above theorem is finite. Sárközy asked whether there exist two infinite sets of nonnegative integers $\mathcal{A}$ and $\mathcal{B}$ with infinite symmetric difference, that is,

$$
|(A \cup B) \backslash(A \cap B)|=\infty
$$

and

$$
R_{2, \mathcal{A}}^{(i)}(n)=R_{2, \mathcal{B}}^{(i)}(n)
$$

if $n \geq n_{0}$, for $i=1,2,3$. For $i=1$, the answer is negative (see [3]). For $i=2$ and 3, respectively, Dombi [3] and Chen and Wang [2] proved that the set of nonnegative integers can be partitioned into two subsets $\mathcal{A}$ and $\mathcal{B}$ such that $R_{2, \mathcal{A}}^{(i)}(n)=R_{2, \mathcal{B}}^{(i)}(n)$ for all $n \geq n_{0}$. In [5] Lev gave a common proof of the above mentioned results of Dombi [3] and Chen and Wang [2]. Using generating functions, Sándor [7] determined the sets $\mathcal{A} \subset \mathbb{N}$ for which either

$$
R_{2, \mathcal{A}}^{(2)}(n)=R_{2, \mathbb{N} \backslash \mathcal{A}}^{(2)}(n) \quad \text { for all } n \geq n_{0}
$$

or

$$
R_{2, \mathcal{A}}^{(3)}(n)=R_{2, \mathbb{N} \backslash \mathcal{A}}^{(3)}(n) \quad \text { for all } n \geq n_{0} .
$$

In [8] Tang gave an elementary proof of Sándor's results and in [1] Chen and Tang studied related questions. We can rewrite Nathanson's theorem in equivalent form as follows.

Equivalent form of Nathanson's Theorem. Let $\mathcal{A}$ and $\mathcal{B}$ be infinite sets of nonnegative integers, $\mathcal{A} \neq \mathcal{B}$. Then $R_{2, \mathcal{A}}^{(1)}(n)=R_{2, \mathcal{B}}^{(1)}(n)$ from a certain point on if and only if there exist positive integers $n_{0}, M$ and finite sets $F_{\mathcal{A}}, F_{\mathcal{B}}, T$ with $F_{\mathcal{A}} \cup F_{\mathcal{B}} \subset$ $\left\{0,1, \ldots, M n_{0}-1\right\}$ and $T \subset\{0,1, \ldots, M-1\}$ such that

$$
\begin{gathered}
\mathcal{A}=F_{\mathcal{A}} \cup\left\{k M+t: k \geq n_{0}, t \in T\right\}, \\
\mathcal{B}=F_{\mathcal{B}} \cup\left\{k M+t: k \geq n_{0}, t \in T\right\},
\end{gathered}
$$

and

$$
\left(1-z^{M}\right) \mid\left(F_{\mathcal{A}}(z)-F_{\mathcal{B}}(z)\right) T(z) .
$$

We conjecture that Nathanson's theorem can be generalised in the following way.

Conjecture. Let $h \geq 2, \mathcal{A}$ and $\mathcal{B}$ be infinite sets of nonnegative integers, $\mathcal{A} \neq \mathcal{B}$. Then $R_{h, \mathcal{A}}^{(1)}(n)=R_{h, \mathcal{B}}^{(1)}(n)$ from a certain point on if and only if there exist positive 
integers $n_{0}, M$ and sets $F_{\mathcal{A}}, F_{\mathcal{B}}$ and $T$ such that $F_{\mathcal{A}} \cup F_{\mathcal{B}} \subset\left\{0,1, \ldots, M n_{0}-1\right\}$, $T \subset\{0,1 \ldots, M-1\}$,

$$
\begin{aligned}
\mathcal{A} & =F_{\mathcal{A}} \cup\left\{k M+t: k \geq n_{0}, t \in T\right\}, \\
\mathcal{B} & =F_{\mathcal{B}} \cup\left\{k M+t: k \geq n_{0}, t \in T\right\},
\end{aligned}
$$

and

$$
\left(1-z^{M}\right)^{h-1} \mid\left(F_{\mathcal{A}}(z)-F_{\mathcal{B}}(z)\right) T(z)^{h-1} .
$$

The next theorem shows the sufficiency of the conjecture.

Theorem 1.1. Let $\mathcal{A}$ and $\mathcal{B}$ be infinite sets of nonnegative integers, $\mathcal{A} \neq \mathcal{B}$. If there exist positive integers $n_{0}, M$ and finite sets $F_{\mathcal{A}}, F_{\mathcal{B}}$ and $T$ with $F_{\mathcal{A}} \cup F_{\mathcal{B}} \subset\{0,1, \ldots$, $\left.M n_{0}-1\right\}, T \subset\{0,1, \ldots, M-1\}$ such that

$$
\begin{aligned}
\mathcal{A} & =F_{\mathcal{A}} \cup\left\{k M+t: k \geq n_{0}, t \in T\right\}, \\
\mathcal{B} & =F_{\mathcal{B}} \cup\left\{k M+t: k \geq n_{0}, t \in T\right\},
\end{aligned}
$$

and

$$
\left(1-z^{M}\right)^{h-1} \mid\left(F_{\mathcal{A}}(z)-F_{\mathcal{B}}(z)\right) T(z)^{h-1}
$$

then $R_{h, \mathcal{A}}^{(1)}(n)=R_{h, \mathcal{B}}^{(1)}(n)$ from a certain point on.

However, we can only prove the conjecture in full in the case $h=3$.

Theorem 1.2. Let $\mathcal{A}$ and $\mathcal{B}$ be infinite sets of nonnegative integers, $\mathcal{A} \neq \mathcal{B}$. Then $R_{3, \mathcal{A}}^{(1)}(n)=R_{3, \mathcal{B}}^{(1)}(n)$ from a certain point on if and only if there exist positive integers $n_{0}$, $M$ and sets $F_{\mathcal{A}}, F_{\mathcal{B}}$ and $T$ with $F_{\mathcal{A}} \cup F_{\mathcal{B}} \subset\left\{0,1, \ldots, M n_{0}-1\right\}, T \subset\{0,1, \ldots, M-1\}$ such that

$$
\begin{aligned}
\mathcal{A} & =F_{\mathcal{A}} \cup\left\{k M+t: k \geq n_{0}, t \in T\right\}, \\
\mathcal{B} & =F_{\mathcal{B}} \cup\left\{k M+t: k \geq n_{0}, t \in T\right\},
\end{aligned}
$$

and

$$
\left(1-z^{M}\right)^{2} \mid\left(F_{\mathcal{A}}(z)-F_{\mathcal{B}}(z)\right) T(z)^{2} .
$$

In 2011, Yang [9] gave another proof of Nathanson's theorem without using generating functions. In his paper he posed the following problem.

Problem. If $p \geq 3$ is a prime and $\mathcal{A}$ is an infinite set of nonnegative integers, then does there exist an infinite set of nonnegative integers $\mathcal{B}$ with $\mathcal{A} \neq \mathcal{B}$ such that $R_{p, \mathcal{A}}^{(1)}(n)=R_{p, \mathcal{B}}^{(1)}(n)$ for all sufficiently large $n$ ?

In this paper we show that the answer to Yang's question is negative.

THEOREM 1.3. For every prime $p$ there exists an infinite set of nonnegative integers $\mathcal{A}$ such that for any infinite set of integers $\mathcal{B}, \mathcal{A} \neq \mathcal{B}$, we have $R_{p, \mathcal{A}}^{(1)}(n) \neq R_{p, \mathcal{B}}^{(1)}(n)$ for infinitely many positive integer $n$. 
We studied some similar problems for the following results.

THEOREM 1.4. For every positive integer $H \geq 2$ there exist infinite sets of nonnegative integers $\mathcal{A}, \mathcal{B}, \mathcal{A} \neq \mathcal{B}$ such that $R_{h, \mathcal{A}}^{(l)}(n)=R_{h, \mathcal{B}}^{(l)}(n)$, for every $l=1,2,3$ and $2 \leq h \leq H$ from a certain point on.

In the special case $l=1$, Theorem 1.4 cannot be extended for infinitely many $h$.

THEOREM 1.5. If for some infinite sets of nonnegative integers $\mathcal{A}$ and $\mathcal{B}$ the representation function $R_{h, \mathcal{A}}^{(1)}(n)=R_{h, \mathcal{B}}^{(1)}(n)$, for $n \geq n_{0}(h)$, for infinitely many positive integers $h \geq 2$, then $\mathcal{A}=\mathcal{B}$.

In this paper let $A(z), B(z), F_{\mathcal{A}}(z), F_{\mathcal{B}}, T(z), S(z)$ denote the generating functions of the sets $\mathcal{A}, \mathcal{B}, F_{\mathcal{A}}, F_{\mathcal{B}}, T$ and $S \subseteq \mathbb{N}$ (that is, $A(z)=\sum_{a \in \mathcal{A}} z^{a}$, where $z$ is a complex number, $z=r \cdot e^{2 \pi i \theta}$, and so on, and these functions converge in the open unit disc).

\section{Proof of Theorem 1.1}

In order to prove Theorem 1.1 we need to show that $A(z)^{h}-B(z)^{h}=P(z)$, where $P(z)$ is a polynomial. By definition of $\mathcal{A}$ and $\mathcal{B}$,

$$
A(z)=F_{\mathcal{A}}(z)+\frac{z^{n_{0} M} T(z)}{1-z^{M}}
$$

and

$$
B(z)=F_{\mathcal{B}}(z)+\frac{z^{n_{0} M} T(z)}{1-z^{M}} .
$$

Therefore, using the binomial theorem,

$$
\begin{aligned}
A(z)^{h}-B(z)^{h} & =\left(F_{\mathcal{A}}(z)+\frac{z^{n_{0} M} T(z)}{1-z^{M}}\right)^{h}-\left(F_{\mathcal{B}}(z)+\frac{z^{n_{0} M} T(z)}{1-z^{M}}\right)^{h} \\
& =\sum_{k=1}^{h}\left(\begin{array}{l}
h \\
k
\end{array}\right)\left(\frac{z^{n_{0} M} T(z)}{1-z^{M}}\right)^{h-k}\left(F_{\mathcal{A}}(z)^{k}-F_{\mathcal{B}}(z)^{k}\right) .
\end{aligned}
$$

Now we verify that, for $1 \leq k \leq h-1$,

$$
\left(1-z^{M}\right)^{h-k} \mid T(z)^{h-k}\left(F_{\mathcal{A}}(z)^{k}-F_{\mathcal{B}}(z)^{k}\right) .
$$

Since

$$
F_{\mathcal{A}}(z)-F_{\mathcal{B}}(z) \mid F_{\mathcal{A}}(z)^{k}-F_{\mathcal{B}}(z)^{k}
$$

it is enough to show that

$$
\left(1-z^{M}\right)^{h-k} \mid T(z)^{h-k}\left(F_{\mathcal{A}}(z)-F_{\mathcal{B}}(z)\right) .
$$

For a given integer $m$, where $m \mid M$, denote by $\Phi_{m}(z)$ the $m$ th cyclotomic polynomial. It remains to prove that

$$
\Phi_{m}(z)^{h-k} \mid T(z)^{h-k}\left(F_{\mathcal{A}}(z)-F_{\mathcal{B}}(z)\right) .
$$


Let $T(z)=\Phi_{m}(z)^{k_{1}} u(z)$ and $F_{\mathcal{A}}(z)-F_{\mathcal{B}}(z)=\Phi_{m}(z)^{k_{2}} v(z)$, where $u(z)$ and $v(z)$ are polynomials with the property $\Phi_{m}(z) \nmid u(z) v(z)$. By assumption of Theorem 1.1 we know that $(h-1) k_{1}+k_{2} \geq h-1$. Thus either $k_{1}=0$, so $k_{2} \geq h-1$ and therefore

$$
\Phi_{m}(z)^{h-k} \mid F_{\mathcal{A}}(z)-F_{\mathcal{B}}(z),
$$

or $k_{1} \geq 1$ and therefore

$$
\Phi_{m}(z)^{h-k} \mid T(z)^{h-k}
$$

which completes the proof.

\section{Proof of Theorem $\mathbf{1 . 2}$}

First we would like to prove that if $R_{3, \mathcal{A}}^{(1)}(n)=R_{3, \mathcal{B}}^{(1)}(n)$ from a certain point on then we have nonnegative integers $n_{0}, M$ and finite sets of nonnegative integers $F_{\mathcal{A}}, F_{\mathcal{B}}$, $T$ with $F_{\mathcal{A}} \cup F_{\mathcal{B}} \subset\left\{0,1, \ldots, M n_{0}-1\right\}, T \subset\{0,1, \ldots, M-1\}$ such that (1.1)-(1.3) hold. It is easy to see that there exists a positive integer $N_{0}$ such that $\mathcal{A} \cap\left[N_{0},+\infty\right)=$ $\mathcal{B} \cap\left[N_{0},+\infty\right)$, because $R_{3, \mathcal{A}}^{(1)}(n) \equiv 0(\bmod 3)$ if $n / 3 \notin \mathcal{A}$, and $R_{3, \mathcal{A}}^{(1)}(n) \equiv 1(\bmod 3)$ if $n / 3 \in \mathcal{A}$. Similarly, $R_{3, \mathcal{B}}^{(1)}(n) \equiv 0(\bmod 3)$ if $n / 3 \notin \mathcal{B}$, and $R_{3, \mathcal{B}}^{(1)}(n) \equiv 1(\bmod 3)$ if $n / 3 \in \mathcal{B}$. Thus there exist an integer $N_{1}$, finite sets of nonnegative integers $F_{\mathcal{A}}$, $F_{\mathcal{B}}$ and an infinite set of nonnegative integers $S$ with $F_{\mathcal{A}}, F_{\mathcal{B}} \subset\left\{0,1, \ldots, N_{1}\right\}, S \subset$ $\left\{N_{1}+1, N_{1}+2, \ldots\right\}$ such that

$$
\mathcal{A}=F_{\mathcal{A}} \cup S
$$

and

$$
\mathcal{B}=F_{\mathcal{B}} \cup S .
$$

Since $A(z)$ and $B(z)$ are the generating functions of the sets $\mathcal{A}$ and $\mathcal{B}$,

$$
A^{3}(z)=\sum_{n=0}^{\infty} R_{3, \mathcal{A}}^{(1)}(n) z^{n}
$$

and

$$
B^{3}(z)=\sum_{n=0}^{\infty} R_{3, \mathcal{B}}^{(1)}(n) z^{n} .
$$

Since $R_{3, \mathcal{A}}^{(1)}(n)=R_{3, \mathcal{B}}^{(1)}(n)$, for $n \geq N_{2}$, it is clear that there is a polynomial $Q(z)$ such that

$$
\sum_{n=1}^{\infty} R_{3, \mathcal{A}}^{(1)}(n) z^{n}-\sum_{n=1}^{\infty} R_{3, \mathcal{B}}^{(1)}(n) z^{n}=Q(z) .
$$

Thus $A^{3}(z)-B^{3}(z)=Q(z)$. In view of (3.1) and (3.2) it follows that

$$
A(z)=F_{\mathcal{A}}(z)+S(z)
$$

and

$$
B(z)=F_{\mathcal{B}}(z)+S(z)
$$


Hence

$$
\begin{array}{r}
\left(S(z)+F_{\mathcal{A}}(z)\right)^{3}-\left(S(z)+F_{\mathcal{B}}(z)\right)^{3}=3 S^{2}(z) F_{\mathcal{A}}(z)+3 S(z) F_{\mathcal{A}}^{2}(z)-3 S^{2}(z) F_{\mathcal{B}}(z) \\
-3 S(z) F_{\mathcal{B}}^{2}(z)+F_{\mathcal{A}}^{3}(z)-F_{\mathcal{B}}^{3}(z)=Q(z) .
\end{array}
$$

Since $F_{\mathcal{A}}$ and $F_{\mathcal{B}}$ are finite sets there is a polynomial $P(z)$ such that

$$
3 S(z)\left(S(z)+F_{\mathcal{A}}(z)+F_{\mathcal{B}}(z)\right)\left(F_{\mathcal{A}}(z)-F_{\mathcal{B}}(z)\right)=P(z) .
$$

It follows that there are relatively prime polynomials $P_{1}(z)$ and $P_{2}(z)$ such that

$$
3 S(z)\left(S(z)+F_{\mathcal{A}}(z)+F_{\mathcal{B}}(z)\right)=\frac{P(z)}{F_{\mathcal{A}}(z)-F_{\mathcal{B}}(z)}=\frac{P_{1}(z)}{P_{2}(z)} .
$$

The left-hand side of (3.4) converges in the open unit disc. Then

$$
F_{\mathcal{A}}(z)-F_{\mathcal{B}}(z)=z^{l}\left(c_{0}+c_{1} z+\cdots+c_{q} z^{q}\right),
$$

where $\left|c_{0}\right|=1$ and $\left|c_{q}\right|=1$. Thus

$$
P_{2}(z)=z^{k}\left(d_{0}+d_{1} z+\cdots+d_{w} z^{w}\right),
$$

where $\left|d_{0}\right|=1$ and $\left|d_{w}\right|=1$. Assume that $k \neq 0$. Then the right-hand side of (3.4) tends to infinity in absolute value and the left-hand side of (3.4) converges in absolute value when $z \rightarrow 0$, which is absurd. So $k=0$. Thus

$$
P_{2}(z)=d_{0}+d_{1} z+\cdots+d_{w} z^{w}
$$

and

$$
F_{\mathcal{A}}(z)-F_{\mathcal{B}}(z)=\sum_{n=0}^{N_{1}} f_{n} z^{n},
$$

where all the $f_{n}$ are integers and $\left|f_{n}\right| \leq 1$.

We now prove the following lemma.

LeMMa 3.1. If $P_{2}\left(z_{0}\right)=0$ for some complex number $z_{0}$, then $\left|z_{0}\right| \geq 1$.

Proof. We prove this by contradiction. Assume that there exists $z_{0} \in \mathbb{C}$ such that $P_{2}\left(z_{0}\right)=0$ and $\left|z_{0}\right|<1$. Take the limit as $z \rightarrow z_{0}$ in (3.4). Then

$$
3 S(z)\left(S(z)+F_{\mathcal{A}}(z)+F_{\mathcal{B}}(z)\right) \rightarrow 3 S\left(z_{0}\right)\left(S\left(z_{0}\right)+F_{\mathcal{A}}\left(z_{0}\right)+F_{\mathcal{B}}\left(z_{0}\right)\right)
$$

and

$$
\left|3 S(z)\left(S(z)+F_{\mathcal{A}}(z)+F_{\mathcal{B}}(z)\right)\right| \rightarrow\left|3 S\left(z_{0}\right)\left(S\left(z_{0}\right)+F_{\mathcal{A}}\left(z_{0}\right)+F_{\mathcal{B}}\left(z_{0}\right)\right)\right| \in \mathbb{R} .
$$

Since $P_{1}(z)$ and $P_{2}(z)$ are relatively prime, $P_{1}\left(z_{0}\right) \neq 0$,

$$
\left|\frac{P_{1}(z)}{P_{2}(z)}\right| \rightarrow \infty
$$

as $z \rightarrow z_{0}$, which is absurd. 
We may suppose that $d_{w}=1$. This means that the roots of $P_{2}(z)$ are algebraic integers. In this case the product of the roots of the polynomial $P_{2}(z)$ is $d_{0}$ and $\left|d_{0}\right|=1$. It follows from Lemma 3.1 that the absolute value of each root is 1 . Since $d_{w}=1$ it is well known that the roots lie with their conjugates in the closed unit disc. It follows from a well-known theorem of Kronecker [4] that every root is a root of unity. Thus

$$
P_{2}(z)=\prod_{j=1}^{u}\left(z-\varepsilon_{j}\right)^{m_{j}}
$$

where $\varepsilon_{j}$ is a root of unity and has multiplicity $m_{j}$.

We prove that for every $j, m_{j} \leq 2$. Assume that there exists an $m_{j} \geq 3$. Then, from (3.4),

$$
3 S(z)\left(S(z)+F_{\mathcal{A}}(z)+F_{\mathcal{B}}(z)\right)\left(z-\varepsilon_{j}\right)^{2}=\frac{P_{1}(z)}{R(z)\left(z-\varepsilon_{j}\right)^{m_{j}-2}},
$$

where $R(z)$ is a polynomial, $R\left(\varepsilon_{j}\right) \neq 0$ and $P_{1}\left(\varepsilon_{j}\right) \neq 0$. Then

$$
\left|\frac{P_{1}\left(r \varepsilon_{j}\right)}{R\left(r \varepsilon_{j}\right)\left(r \varepsilon_{j}-\varepsilon_{j}\right)^{m_{j}-2}}\right| \rightarrow \infty
$$

as $r \rightarrow 1^{-}$. For $z=r \varepsilon_{j}$, we have $\left|z-\varepsilon_{j}\right|^{2}=\left|r \varepsilon_{j}-\varepsilon_{j}\right|^{2}=(1-r)^{2}$ and

$$
S(z)=\sum_{n=0}^{\infty} \chi_{S}(n) z^{n}
$$

where $\chi_{S}(n)$ is the characteristic function of the set $S$ (that is, $\chi_{S}(n)=1$, if $n \in S$ and $\chi_{S}=0$, if $\left.n \notin S\right)$. Then we have the following estimation of the left-hand side of (3.5) for $r<1$ :

$$
\begin{aligned}
& \left|3 S\left(r \varepsilon_{j}\right)\right| \cdot\left|\left(S\left(r \varepsilon_{j}\right)+F_{\mathcal{A}}\left(r \varepsilon_{j}\right)+F_{\mathcal{B}}\left(r \varepsilon_{j}\right)\right)\right| \cdot\left|r \varepsilon_{j}-\varepsilon_{j}\right|^{2} \\
& \quad \leq 3\left(\sum_{n=0}^{\infty} \chi(n)|r|^{n}\right)\left(\sum_{n=0}^{\infty} \chi(n)|r|^{n}+C_{1}\right) \cdot(1-r)^{2} \\
& \quad<\frac{C_{2}}{(1-r)^{2}} \cdot(1-r)^{2}=C_{2},
\end{aligned}
$$

which is absurd.

Thus for some positive integer $M$ we have $P_{2}(z) \mid\left(1-z^{M}\right)^{2}$, so there is a polynomial $P_{3}(z)$ such that

$$
3 S(z)\left(S(z)+F_{\mathcal{A}}(z)+F_{\mathcal{B}}(z)\right)=\frac{P_{3}(z)}{\left(1-z^{M}\right)^{2}} .
$$

Multiplying (3.6) by 12 and adding $9\left(F_{\mathcal{A}}(z)+F_{\mathcal{B}}(z)\right)^{2}$ to it gives us

$$
\left(6 S(z)+3 F_{\mathcal{A}}(z)+3 F_{\mathcal{B}}(z)\right)^{2}=\frac{P_{4}(z)}{\left(1-z^{M}\right)^{2}} .
$$


So

$$
\left(6 S(z)+3 F_{\mathcal{A}}(z)+3 F_{\mathcal{B}}(z)\right)^{2}\left(1-z^{M}\right)^{2}=P_{4}(z) .
$$

We prove that $P_{4}(z)=(u(z))^{2}$, where $u(z)$ is a polynomial with integer coefficients.

Let

$$
\left|\left(6 S(z)+F_{\mathcal{A}}(z)+F_{\mathcal{B}}(z)\right)^{2}\right| \cdot\left|\left(1-z^{M}\right)^{2}\right|=\left|\sum_{n=0}^{\infty} g_{n} z^{n}\right|^{2}=\left|P_{4}(z)\right|,
$$

where $g_{n} \in \mathbb{Z}$. Since $P_{4}(z)$ is a polynomial, the integral $\int_{0}^{2 \pi}\left|P_{4}(z)\right| d \theta$ is bounded for $r \leq 1$. On the other hand, if there exist infinitely many $n$ such that $g_{n} \neq 0$, that is, $g_{n}^{2} \geq 1$, then, using the Parseval formula,

$$
\int_{0}^{2 \pi}\left|\sum_{n=0}^{\infty} g_{n} z^{n}\right|^{2} d \theta=\sum_{n=0}^{\infty} g_{n}^{2} r^{2 n} \rightarrow \infty
$$

as $r \rightarrow 1^{-}$, which is absurd. Thus the series $\sum_{n=0}^{\infty} g_{n} z^{n}=u(z)$ is a polynomial.

This means that there is an integer $K$ such that if $n \geq K$ then $g_{n}=0$, and according to (3.7) if $n \geq N_{3}$ then $g_{n}=6(\chi(n)-\chi(n+M))=0$. So $\chi$ is periodic in $M$. Therefore, there exist a positive integer $n_{0}$ and finite sets $F_{\mathcal{A}}, F_{\mathcal{B}}, T$ with $F_{\mathcal{A}} \cup F_{\mathcal{B}} \subset$ $\left\{0,1, \ldots, M n_{0}-1\right\}$ and $T \subset\{0,1, \ldots, M-1\}$ such that

$$
A=F_{\mathcal{A}} \cup\left\{k M+t: k \geq n_{0}, t \in T\right\},
$$

and

$$
B=F_{\mathcal{B}} \cup\left\{k M+t: k \geq n_{0}, t \in T\right\} .
$$

Hence the generating functions of $\mathcal{A}$ and $\mathcal{B}$ are

$$
A(z)=F_{\mathcal{A}}(z)+\frac{T(z) z^{n_{0} M}}{1-z^{M}}
$$

and

$$
B(z)=F_{\mathcal{B}}(z)+\frac{T(z) z^{n_{0} M}}{1-z^{M}}
$$

Then, from (3.3),

$$
A^{3}(z)-B^{3}(z)=\left(\frac{T(z) z^{n_{0} M}}{1-z^{M}}+F_{\mathcal{A}}(z)\right)^{3}-\left(\frac{T(z) z^{n_{0} M}}{1-z^{M}}+F_{\mathcal{B}}(z)\right)^{3}=Q(z) .
$$

Thus

$$
\frac{3 T(z) z^{n_{0} M}}{1-z^{M}}\left(\frac{T(z) z^{n_{0} M}}{1-z^{M}}+F_{\mathcal{A}}(z)+F_{\mathcal{B}}(z)\right)\left(F_{\mathcal{A}}(z)-F_{\mathcal{B}}(z)\right)=P(z),
$$

that is,

$$
\frac{T(z) z^{n_{0} M}\left(T(z) z^{n_{0} M}+\left(F_{\mathcal{A}}(z)+F_{\mathcal{B}}(z)\right)\left(1-z^{M}\right)\right)\left(F_{\mathcal{A}}(z)-F_{\mathcal{B}}(z)\right)}{\left(1-z^{M}\right)^{2}}=R(z),
$$


where $R(z)$ is also a polynomial. Since $\left(1-z^{M}, z^{n_{0} M}\right)=1$,

$$
\left(1-z^{M}\right)^{2} \mid T(z)\left(T(z) z^{n_{0} M}+\left(F_{\mathcal{A}}(z)+F_{\mathcal{B}}(z)\right)\left(1-z^{M}\right)\right)\left(F_{\mathcal{A}}(z)-F_{\mathcal{B}}(z)\right)
$$

that is,

$$
\begin{aligned}
\left(1-z^{M}\right)^{2} \mid & z^{n_{0} M}\left(F_{\mathcal{A}}(z)-F_{B}(z)\right) T(z)^{2} \\
& +\left(1-z^{M}\right)\left(F_{\mathcal{A}}(z)+F_{B}(z)\right)\left(F_{\mathcal{A}}(z)-F_{B}(z)\right) T(z) .
\end{aligned}
$$

We prove that $1-z^{M} \mid\left(F_{\mathcal{A}}(z)-F_{\mathcal{B}}(z)\right) T(z)$. By way of contradiction, assume that

$$
1-z^{M} \nmid\left(F_{\mathcal{A}}(z)-F_{\mathcal{B}}(z)\right) T(z) .
$$

This means that there exists an integer $k$ such that $k \mid M$ and

$$
\Phi_{k}(z) \nmid\left(F_{\mathcal{A}}(z)-F_{\mathcal{B}}(z)\right) T(z) .
$$

Then, by (3.8),

$$
\Phi_{k}(z) \mid T(z) z^{n_{0} M}+\left(F_{\mathcal{A}}(z)+F_{\mathcal{B}}(z)\right)\left(1-z^{M}\right) .
$$

Thus $\Phi_{k}(z) \mid T(z) z^{n_{0} M}$, but since $\left(\Phi_{k}(z), z^{n_{0} M}\right)=1$ we get $\Phi_{k}(z) \mid T(z)$, which is absurd. Then

$$
\left(1-z^{M}\right)^{2} \mid\left(1-z^{M}\right)\left(F_{\mathcal{A}}(z)+F_{B}(z)\right)\left(F_{\mathcal{A}}(z)-F_{B}(z)\right) T(z),
$$

so, by (3.9),

$$
\left(1-z^{M}\right)^{2} \mid z^{n_{0} M}\left(F_{\mathcal{A}}(z)-F_{B}(z)\right) T(z)^{2} .
$$

But, using the fact that $\left(\left(1-z^{M}\right)^{2}, z^{n_{0} M}\right)=1$, this means that (1.3) holds, as desired.

The other direction is a corollary of Theorem 1.1.

\section{Proof of Theorem 1.3}

Let $\mathcal{A}$ be a sparse set, which means that $\alpha(N)<N^{1 / p}$ (here, $\left.\alpha(N)=|[0, N] \cap \mathcal{A}|\right)$. Let $\mathcal{A}=\left\{a_{1}, a_{2}, \ldots\right\}$. We prove the theorem by contradiction. Assume that $\mathcal{A}, \mathcal{B}$ are different sets and $R_{p, \mathcal{A}}^{(1)}(n)=R_{p, \mathcal{B}}^{(1)}(n)$ from a certain point on. Since $\alpha\left(a_{k}\right)=k<a_{k}^{1 / p}$, it follows that $a_{k}>k^{p}$. The generating function of $\mathcal{A}$ is

$$
\begin{aligned}
A(r) & =\sum_{a \in \mathcal{A}} r^{a}=\sum_{n=0}^{\infty} \chi_{\mathcal{A}}(n) r^{n}=\sum_{n=0}^{\infty}(\alpha(n)-\alpha(n-1)) r^{n} \\
& =\sum_{n=1}^{\infty} \alpha(n)\left(r^{n}-r^{n+1}\right)=(1-r) \sum_{n=0}^{\infty} \alpha(n) r^{n} \\
& =O\left((1-r) \cdot(1-r)^{-1 / p-1}\right)=O\left((1-r)^{-1 / p}\right),
\end{aligned}
$$

as $r \rightarrow 1^{-}$, where $\chi_{\mathcal{A}}(n)$ is the characteristic function of the set $\mathcal{A}$. 
Since $R_{p, \mathcal{A}}^{(1)}(n)=R_{p, \mathcal{B}}^{(1)}(n)$, it is clear that there is a polynomial $P(r)$ such that

$$
A^{p}(r)-B^{p}(r)=P(r)
$$

It is easy to see that there exists a positive integer $N_{0}$ such that $\mathcal{A} \cap\left[N_{0},+\infty\right)=$ $\mathcal{B} \cap\left[N_{0},+\infty\right)$, because $R_{p, \mathcal{A}}^{(1)}(n) \equiv 0(\bmod p)$ if $n / p \notin \mathcal{A}$, and $R_{p, \mathcal{A}}^{(1)}(n) \equiv 1(\bmod p)$ if $n / p \in \mathcal{A}$. Similarly, $R_{p, \mathcal{B}}^{(1)}(n) \equiv 0(\bmod p)$ if $n / p \notin \mathcal{B}$, and $R_{p, \mathcal{B}}^{(1)}(n) \equiv 1(\bmod p)$ if $n / p \in \mathcal{B}$. Thus $A(r)$ differs from $B(r)$ in a polynomial, which means that

$$
B(r)=O\left((1-r)^{-1 / p}\right)
$$

as $r \rightarrow 1^{-}$, as well. So

$$
(A(r)-B(r))\left(A^{p-1}(r)+\cdots+B^{p-1}(r)\right)=P(r) .
$$

Therefore, there exist relatively prime polynomials $R(r)$ and $S(r)$ such that

$$
R(r)\left(A^{p-1}(r)+\cdots+B^{p-1}(r)\right)=S(r) .
$$

As $r \rightarrow 1^{-}$in (4.3) we get that $S(r)$ and $R(r)$ are bounded, and

$$
A^{p-1}(r)+\cdots+B^{p-1}(r) \rightarrow \infty
$$

Therefore $r=1$ must be a root of $R(r)$. Thus

$$
R(r)=(1-r) Q(r) .
$$

Now we can write (4.4) in the form

$$
(1-r) Q(r)\left(A^{p-1}(r)+\cdots+B^{p-1}(r)\right)=S(r) .
$$

Since $Q(r)$ is a polynomial, it is bounded. It follows from (4.1) and (4.2) that

$$
A^{p-1}(r)+\cdots+B^{p-1}(r)=O\left((1-r)^{-(p-1) / p}\right) .
$$

So the order of the left-hand side of $(4.5)$ is $O\left((1-r)^{1 / p}\right)$, as $r \rightarrow 1^{-}$. This means that $S(r)$ tends to zero as $r \rightarrow 1^{-}$. So $S(r)=(1-r) T(r)$, and this contradicts $(R(r), S(r))=1$.

\section{Proof of Theorem 1.4}

The construction of the sets $\mathcal{A}$ and $\mathcal{B}$ is as follows. Let $n$ be a positive integer. Take the binary representation of $n$ to be

$$
n=\sum_{i=0}^{\left\lfloor\log _{2}(n)\right\rfloor} \beta_{i} 2^{i},
$$


where $\beta_{i}=0$ or 1 . Denote by $\operatorname{Bin}(n)=\sum_{i=0}^{\left\lfloor\log _{2}(n)\right\rfloor} \beta_{i}$ the number of ones in the binary representation of $n$. Let

$$
F_{\mathcal{A}}:=\left\{k H !: 0 \leq k<2^{H}, \operatorname{Bin}(k H !) \equiv 0(\bmod 2)\right\}
$$

and

$$
F_{\mathcal{B}}:=\left\{k H !: 0 \leq k<2^{H}, \operatorname{Bin}(k H !) \equiv 1(\bmod 2)\right\} .
$$

We will show that the sets

$$
A=F_{\mathcal{A}} \cup\left\{H ! 2^{H}, H ! 2^{H}+1, \ldots\right\}
$$

and

$$
B=F_{\mathcal{B}} \cup\left\{H ! 2^{H}, H ! 2^{H}+1, \ldots\right\}
$$

are suitable. Let $h$ be a fixed integer, $2 \leq h \leq H$. Then

$$
F_{\mathcal{A}}(z)-F_{\mathcal{B}}(z)=\prod_{i=0}^{H-1}\left(1-z^{H ! 2^{i}}\right),
$$

and therefore

$$
\left(1-z^{h !}\right) \cdots\left(1-z^{2^{h-1} h !}\right) \mid F_{\mathcal{A}}(z)-F_{\mathcal{B}}(z) .
$$

Hence

$$
(1-z) \cdots\left(1-z^{h-1}\right)\left(1-z^{h}\right) \mid F_{\mathcal{A}}(z)-F_{\mathcal{B}}(z) .
$$

The generating function of $R_{h, \mathcal{A}}^{(l)}(n), l=1,2,3$, can be written using a sieve formula with suitable real numbers $C_{k_{1}, \ldots, k_{h}}$ :

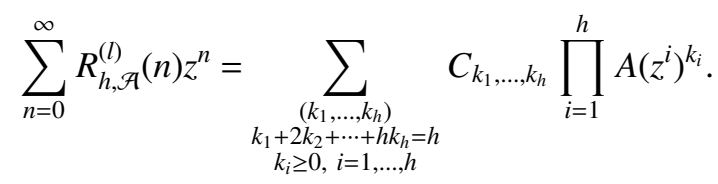

We would like to prove that there is a polynomial $P(z)$ such that

$$
\sum_{n=0}^{\infty} R_{h, \mathcal{A}}^{(l)}(n) z^{n}-\sum_{n=0}^{\infty} R_{h, \mathcal{B}}^{(l)}(n) z^{n}=P(z) .
$$

From (5.1), the left-hand side of (5.2) is equivalent to

$$
\sum_{\substack{\left(k_{1}, \ldots, k_{h}\right) \\ k_{1}+2 k_{2}+\cdots+h k_{h}=h \\ k_{i} \geq 0, i=1, \ldots, h}} C_{k_{1}, \ldots, k_{h}}\left(\prod_{i=1}^{h} A\left(z^{i}\right)^{k_{i}}-\prod_{i=1}^{h} B\left(z^{i}\right)^{k_{i}}\right) .
$$

In view of

$$
A(z)=F_{\mathcal{A}}(z)+\frac{z^{H ! 2^{H}}}{1-z}
$$


and

$$
B(z)=F_{\mathcal{B}}(z)+\frac{z^{H ! 2^{H}}}{1-z}
$$

we get that (5.3) is equivalent to

$$
\sum_{\substack{\left(k_{1}, \ldots, k_{h}\right) \\ k_{1}+2 k_{2}+\cdots+h k_{h}=h \\ k_{i} \geq 0, i=1, \ldots, h}} C_{k_{1}, \ldots, k_{h}}\left(\prod_{i=1}^{h}\left(F_{\mathcal{A}}\left(z^{i}\right)+\frac{z^{i H ! 2^{H}}}{1-z^{i}}\right)^{k_{i}}-\prod_{i=1}^{h}\left(F_{\mathcal{B}}\left(z^{i}\right)+\frac{z^{i H ! 2^{H}}}{1-z^{i}}\right)^{k_{i}}\right) .
$$

It is enough to show that the difference of the products in (5.4) is a polynomial for every $h$-tuple $\left(k_{1}, \ldots, k_{h}\right)$. Let the $h$-tuple $\left(k_{1}, \ldots, k_{h}\right)$ be fixed. Using the binomial theorem, we get that for suitable constants $D_{j_{1}, \ldots, j_{h}}$ this expression is equal to

$$
\begin{aligned}
\left(\prod_{i=1}^{h} \sum_{j_{i}=0}^{k_{i}}\left(\begin{array}{c}
k_{i} \\
j_{i}
\end{array}\right)\left(F_{\mathcal{A}}\left(z^{i}\right)\right)^{j_{i}}\left(\frac{z^{i H ! 2^{H}}}{1-z^{i}}\right)^{k_{i}-j_{i}}\right)-\left(\prod_{i=1}^{h} \sum_{j_{i}=0}^{k_{i}}\left(\begin{array}{c}
k_{i} \\
j_{i}
\end{array}\right)\left(F_{\mathcal{B}}\left(z^{i}\right)\right)^{j_{i}}\left(\frac{z^{i H ! 2^{H}}}{1-z^{i}}\right)^{k_{i}-j_{i}}\right) \\
=\sum_{\substack{\left(j_{1}, \ldots, j_{h}\right) \\
0 \leq j_{i} \leq k_{i}, i=1, \ldots, h}} D_{j_{1}, \ldots, j_{h}}\left(\prod_{i=1}^{h}\left(\frac{z^{i H ! 2^{H}}}{1-z^{i}}\right)^{k_{i}-j_{i}}\right)\left(\prod_{i=1}^{h}\left(F_{\mathcal{A}}\left(z^{i}\right)\right)^{j_{i}}-\prod_{i=1}^{h}\left(F_{\mathcal{B}}\left(z^{i}\right)\right)^{j_{i}}\right) .
\end{aligned}
$$

We will show that

$$
\left(\prod_{i=1}^{h}\left(\frac{z^{i H ! 2^{H}}}{1-z^{i}}\right)^{k_{i}-j_{i}}\right)\left(\prod_{i=1}^{h}\left(F_{\mathcal{A}}\left(z^{i}\right)\right)^{j_{i}}-\prod_{i=1}^{h}\left(F_{\mathcal{B}}\left(z^{i}\right)\right)^{j_{i}}\right)
$$

is a polynomial. To show this we will prove that there is a polynomial $Q(z)$ such that

$$
\prod_{i=1}^{h}\left(\frac{z^{i H ! 2^{H}}}{1-z^{i}}\right)^{k_{i}-j_{i}}=\frac{Q(z)}{(1-z) \cdots\left(1-z^{h-1}\right)\left(1-z^{h}\right)},
$$

and

$$
(1-z) \cdots\left(1-z^{h-1}\right)\left(1-z^{h}\right) \mid \prod_{i=1}^{h}\left(F_{\mathcal{A}}\left(z^{i}\right)\right)^{j_{i}}-\prod_{i=1}^{h}\left(F_{\mathcal{B}}\left(z^{i}\right)\right)^{j_{i}} .
$$

To deduce (5.5) it is enough to show that

$$
\prod_{i=1}^{h}\left(1-z^{i}\right)^{k_{i}-j_{i}} \mid(1-z) \cdots\left(1-z^{h-1}\right)\left(1-z^{h}\right) .
$$

A root of the product $\prod_{i=1}^{h}\left(1-z^{i}\right)^{k_{i}-j_{i}}$ is a primitive $i$ th root of unity, for some $i \leq h$. Let $\varepsilon_{i}$ denote a primitive $i$ th root of unity. The multiplicity of $\varepsilon_{i}$ in the polynomial $(1-z) \cdots\left(1-z^{h-1}\right)\left(1-z^{h}\right)$ is $\lfloor h / i\rfloor$. The multiplicity of $\varepsilon_{i}$ in the polynomial $\prod_{i=1}^{h}\left(1-z^{i}\right)^{k_{i}-j_{i}}$ is

$$
\left(k_{i}-j_{i}\right)+\left(k_{2 i}-j_{2 i}\right)+\cdots \leq k_{i}+k_{2 i}+\cdots
$$


We know that $k_{1}+2 k_{2}+\cdots+h k_{h}=h$. Therefore,

$$
i k_{i}+i k_{2 i}+\cdots \leq i k_{i}+2 i k_{2 i}+\cdots \leq 1 k_{1}+2 k_{2}+\cdots+h k_{h}=h .
$$

This means that

$$
k_{i}+k_{2 i}+\cdots \leq\left\lfloor\frac{h}{i}\right\rfloor
$$

which proves (5.5).

It remains to prove the following lemma, which verifies (5.6).

Lemma 5.1. If $(1-z) \cdots\left(1-z^{h-1}\right)\left(1-z^{h}\right) \mid F_{\mathcal{A}}(z)-F_{\mathcal{B}}(z)$ then, for all $t$-tuples $\left(l_{1}, \ldots, l_{t}\right)$,

$$
(1-z) \cdots\left(1-z^{h-1}\right)\left(1-z^{h}\right) \mid \prod_{i=1}^{t}\left(F_{\mathcal{A}}\left(z^{i}\right)\right)^{l_{i}}-\prod_{i=1}^{t}\left(F_{\mathcal{B}}\left(z^{i}\right)\right)^{l_{i}} .
$$

Proof. We prove this result by induction on $t$. If $t=1$ then we show that

$$
(1-z) \cdots\left(1-z^{h-1}\right)\left(1-z^{h}\right) \mid\left(F_{\mathcal{A}}(z)\right)^{l_{1}}-\left(F_{\mathcal{B}}(z)\right)^{l_{1}} .
$$

Since

$$
\left(F_{\mathcal{A}}(z)\right)^{l_{1}}-\left(F_{\mathcal{B}}(z)\right)^{l_{1}}=\left(F_{\mathcal{A}}(z)-F_{\mathcal{B}}(z)\right)\left(\left(F_{\mathcal{A}}(z)\right)^{l_{1}-1}+\cdots+\left(F_{\mathcal{B}}(z)\right)^{l_{1}-1}\right),
$$

we get that the case $t=1$ holds.

Now assume that the lemma holds for all $t$ or less. For $t+1$ we need to show that

$$
(1-z) \cdots\left(1-z^{h-1}\right)\left(1-z^{h}\right) \mid \prod_{i=1}^{t+1}\left(F_{\mathcal{A}}\left(z^{i}\right)\right)^{l_{i}}-\prod_{i=1}^{t+1}\left(F_{\mathcal{B}}\left(z^{i}\right)\right)^{l_{i}} .
$$

The right-hand side of (5.7) is equal to

$$
\begin{aligned}
\left(F_{\mathcal{A}}(z)\right)^{l_{1}} \cdots\left(F_{\mathcal{A}}\left(z^{t+1}\right)^{l_{t+1}}\right)-\left(F_{\mathcal{A}}(z)\right)^{l_{1}} \cdots\left(F_{\mathcal{A}}\left(z^{t}\right)\right)^{l_{t}}\left(F_{\mathcal{B}}\left(z^{t+1}\right)\right)^{l_{t+1}} \\
+\left(F_{\mathcal{A}}(z)\right)^{l_{1}} \cdots\left(F_{\mathcal{A}}\left(z^{t}\right)\right)^{l_{t}}\left(F_{\mathcal{B}}\left(z^{t+1}\right)\right)^{l_{t+1}}-\left(F_{\mathcal{B}}(z)\right)^{l_{1}} \cdots\left(F_{\mathcal{B}}\left(z^{t+1}\right)\right)^{l_{t+1}} \\
=\left(F_{\mathcal{A}}(z)\right)^{l_{1}} \cdots\left(F_{\mathcal{A}}\left(z^{t}\right)\right)^{l_{t}}\left(\left(F_{\mathcal{A}}\left(z^{t+1}\right)\right)^{l_{t+1}}-\left(F_{\mathcal{B}}\left(z^{t+1}\right)\right)^{l_{t+1}}\right) \\
\quad-\left(F_{\mathcal{B}}\left(z^{t+1}\right)\right)^{l_{t+1}}\left(\left(F_{\mathcal{A}}(z)\right)^{l_{1}} \cdots\left(F_{\mathcal{A}}\left(z^{t}\right)\right)^{l_{t}}-\left(F_{\mathcal{B}}(z)\right)^{l_{1}} \cdots\left(F_{\mathcal{B}}\left(z^{t}\right)\right)^{l_{t}}\right) .
\end{aligned}
$$

Because of our assumption, the second term is divisible by $(1-z) \cdots\left(1-z^{h-1}\right)$. $\left(1-z^{h}\right)$. Since

$$
(1-z) \cdots\left(1-z^{h-1}\right)\left(1-z^{h}\right) \mid\left(1-z^{t+1}\right) \cdots\left(1-z^{h(t+1)}\right)
$$

and

$$
\left(1-z^{t+1}\right) \cdots\left(1-z^{h(t+1)}\right) \mid\left(F_{\mathcal{A}}\left(z^{t+1}\right)\right)^{l_{t+1}}-\left(\left(F_{\mathcal{B}}\left(z^{t+1}\right)\right)^{l_{t+1}}\right),
$$

this completes the induction. 


\section{Proof of Theorem 1.5}

We prove the theorem by contradiction. Assume that for infinite sets of nonnegative integers $\mathcal{A}, \mathcal{B}, \mathcal{A} \neq \mathcal{B}$, there is an infinite sequence of integers $2 \leq h_{1}<h_{2}<\cdots<h_{i}<$ $\cdots$ and polynomials $P_{i}(r)$ such that

$$
A^{h_{i}}(r)-B^{h_{i}}(r)=\sum_{n=0}^{\infty}\left(R_{h_{i}, \mathcal{A}}^{(1)}(n)-R_{h_{i}, \mathcal{B}}^{(1)}(n)\right) r^{n}=P_{i}(r) .
$$

Then

$$
P_{i}(r)=A^{h_{i}}(r)-B^{h_{i}}(r)=(A(r)-B(r))\left(A^{h_{i}-1}(r)+A^{h_{i}-2}(r) B(r)+\cdots+B^{h_{i}-1}(r)\right) .
$$

As $r \rightarrow 1^{-}$,

$$
\begin{aligned}
\frac{P_{i+1}(r)}{P_{i}(r)} & =\frac{A^{h_{i}-1}(r)+A^{h_{i}-2}(r) B(r)+\cdots+B^{h_{i}-1}(r)}{A^{h_{i+1}-1}(r)+A^{h_{i+1}-2}(r) B(r)+\cdots+B^{h_{i+1}-1}(r)} \\
& \leq \frac{h_{i} \cdot \max \left\{A^{h_{i}-1}(r), B^{h_{i}-1}(r)\right\}}{\max \left\{A^{h_{i+1}-1}(r), B^{h_{i+1}-1}(r)\right\}} \rightarrow 0 .
\end{aligned}
$$

Let $P_{i}(r)=(1-r)^{m_{i}} Q_{i}(r)$, where $m_{i}$ is a nonnegative integer, $Q_{i}(r)$ is a polynomial and $Q_{i}(1) \neq 0$. Thus

$$
\frac{P_{i+1}(r)}{P_{i}(r)}=\frac{(1-r)^{m_{i+1}} Q_{i+1}(r)}{(1-r)^{m_{i}} Q_{i}(r)},
$$

and $m_{i+1}<m_{i}$. We get that $m_{1}>m_{2}>\cdots$, which is absurd.

\section{References}

[1] Y.-G. Chen and M. Tang, 'Partitions of natural numbers with the same representation functions', J. Number Theory 129 (2009), 2689-2695.

[2] Y.-G. Chen and B. Wang, 'On additive properties of two special sequences', Acta Arith. 110.3 (2003), 299-303.

[3] G. Dombi, 'Additive properties of certain sets', Acta Arith. 103.2 (2002), 137-146.

[4] L. Kronecker, 'Zwei Sätze über Gleichungen mit ganzzahligen Coefficienten', J. reine angew. Math. 53 (1857), 173-175.

[5] V. F. Lev, 'Reconstructing integer sets from their representation functions', Electr. J. Combin. 11 (2004), R78.

[6] M. B. Nathanson, 'Representation functions of sequences in additive number theory', Proc. Amer. Math. Soc. 72 (1978), 16-20.

[7] Cs. Sándor, 'Partitions of natural numbers and their representation functions', Integers 4 (2004), A18.

[8] M. Tang, 'Partitions of the set of natural numbers and their representation functions', Discrete Math. 308 (2008), 2614-2616.

[9] Q.-H. Yang, 'Another proof of Nathanson's theorems', J. Integer Sequences 14 (2011). 
SÁNDOR Z. KISS, Institute of Mathematics,

Budapest University of Technology and Economics, H-1529 Budapest, PO Box 91, Hungary

and

Computer and Automation Research Institute of the Hungarian Academy of Sciences, Lágymányosi utca 11, H-1111 Budapest, Hungary

e-mail: kisspest@cs.elte.hu

ESZTER ROZGONYI, Institute of Mathematics,

Budapest University of Technology and Economics, H-1529 Budapest, PO Box 91, Hungary

e-mail: reszti@math.bme.hu

CSABA SÁNDOR, Institute of Mathematics,

Budapest University of Technology and Economics, H-1529 Budapest, PO Box 91, Hungary

e-mail: csandor@math.bme.hu 